\title{
Sepsis - eine Herausforderung für die Medizin und das Gesundheitswesen
}

W enn der Körper innerlich heiß und außen kalt ist, dann verläuft die Krankheit tödlich“, so beschrieb bereits Hippokrates den Verlauf einer Sepsis, ohne die Kenntnisse der modernen Mikrobiologie und Immunologie zu haben. Ausgelöst durch Bakterien, Pilze oder Parasiten bedroht die Sepsis die Menschheit seit Jahrtausenden, lange vor dem Auftreten der Zivilisationskrankheiten wie Diabetes oder der koronaren Herzkrankheit. Doch trotz aller Fortschritte, die wir in der Aufklärung der Pathophysiologie der Sepsis gemacht haben, und trotz all unserer Bemühungen versterben noch immer etwa 40\% der Patienten - obwohl sie eine Antibiotikatherapie und eine Intensivbehandlung erhalten.

Die Sepsis gefährdet in vielen medizinischen Fachdisziplinen die Therapieerfolge der modernen Medizin: Sie ist eine potenziell tödliche Komplikation nach großen Operationen oder im Rahmen einer Chemotherapie bei Tumorerkrankungen. Aber auch gesunde Menschen erkranken an Sepsis, am häufigsten ausgelöst durch eine Lungenentzündung.

Ab dem 1. Januar 2004 sind die G-DRGs (,german diagnosis related groups) die alleinige Abrechnungsgrundlage aller Krankenhäuser in Deutschland. Der ICD10 GM erhält hierdurch als Grundlage der G-DRGs eine neue Bedeutung. Seine gegenwärtige Fassung fasst den Begriff der „Sepsis“ jedoch durch die Anlehnung an eine rein mikrobiologisch orientierte Definition der Erkrankung extrem weit und unscharf.

Da in der Behandlung der Sepsis - ähnlich wie in der Behandlung des akuten Herzinfarktes - jede Minute zählt, ist ihre frühzeitige Erkennung und damit eine verbesserte Diagnostik entscheidend. Nach jüngsten Datenerhebungen aus den USA erkranken pro Jahr 751000 Patienten an Sepsis, mit steigender Tendenz in den letzten vier Jahren. Das entspricht einer Inzidenz von 300 SepsisFällen pro 100000 Einwohner. Im Vergleich dazu liegt die Inzidenz von Dickdarmkrebs bei 50, von Brustkrebs bei 110 und von AIDS bei 17 pro 100000 Einwohner.

Die Kenntnisse der Öffentlichkeit über diese Erkrankung sind jedoch sehr gering. In einer jüngsten repräsentativen Umfrage in Europa und den USA zeigte sich, dass weniger als 15\% der Befragten den Begriff „Sepsis“ kannten. In Deutschland brachten immerhin 50\% die Sepsis mit einer Infektion in Verbindung. Eine der Ursachen hierfür ist, dass in der Kommunikation zwischen Arzt und Patient bzw. den Angehörigen und in den Todesstatistiken lediglich die auslösende Infektion, also beispielsweise die Lungen- oder Bauchfellentzündung, jedoch nicht die Todesursache, nämlich die Sepsis, benannt wird. Die Sepsis ist jedoch auch eine beträchtliche sozioökonomische Herausforderung, in Deutschland betragen allein die direkten Kosten für die Sepsisbehandlung ungefähr 2,25 Milliarden Euro jährlich.
Erfreulicherweise hat nach Jahrzehnten der Stagnation in den letzten drei Jahren die Sepsisforschung große Fortschritte gemacht, die nun direkt in die Versorgung der Patienten umgesetzt werden müssen. In klinischen Studien konnte durch eine niedrig dosierte Hydrokortisontherapie, eine zielgerichtete und optimierte Kreislauftherapie sowie durch die Anwendung von aktiviertem Protein C die Sterblichkeit um bis zu 15\% gesenkt werden. Eine strikte Kontrolle des Blutzuckers verringert bei Krankenhauspatienten das Risiko, an einer Sepsis zu erkranken.

Seit Februar 2001 führt ein vom Bundesministerium für Bildung und Forschung gefördertes Kompetenznetzwerk Sepsis („SepNet“) epidemiologische und klinische Studien in Deutschland durch. Ziel dieses Sepsisnetzwerkes und der ebenfalls 2001 gegründeten Deutschen Sepsis-Gesellschaft e.V. ist es, die Sterblichkeit an der Sepsis um 25\% zu senken. Die Beiträge in diesem Heft des klinikarzt sollen dazu dienen, das Wissen um diese hinsichtlich ihrer Häufigkeit und Gefährlichkeit extrem unterschätzte Erkrankung zu vertiefen, bzw. dazu aufrufen, dieses Wissen in die Behandlung im Sinne unserer Patienten aktiv und nachhaltig einfließen zu lassen.

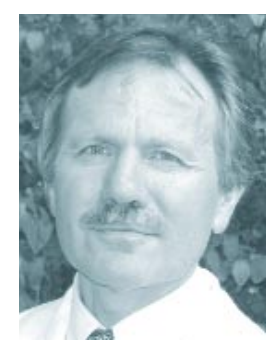

Prof. Dr. K. Reinhart, Jena (Gasteditor)

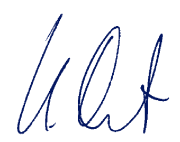

